\title{
N-TERMINAL PRO-B-TYPE NATRIURETIC PEPTIDE-PROGHSTIC VALUE FOR DEATH WITHIN 24 HOURS IN SEVERE POLYTRAUMA PATIENTS WITH CHEST TRAUMA
}

\section{Tase Ghinguleac Gristina1, Tudoran Rodica1, Ghirila Sergiu², Gurgas Leonard², Ion lleana²}

${ }^{1}$ County Clinical Emergency Hospital of Constanta

${ }^{2}$ Faculty of Medicine, University "Ovidius" of Constanta

\begin{abstract}
Patients with multiple trauma with thoracic trauma have higher death rates compared to multiple trauma patients without thoracic trauma, mainly because of cardiac injury. We investigated the 24 hours prognostic value of NT-proB-type Natriuretic Peptide (Nt-ProBNP) in polytraumatised patients with thoracic trauma.

The study group was composed of 33 patients, 25 males and 8 females. During the study, the endpoint, which was death in the first 24 hours after admission was observed in 33\% of the patients (11 patients).

Using a cut-off point of $125 \mathrm{pg} / \mathrm{ml}$, the sensitivity of the test was $100 \%$ and the specificity was $59.09 \%$.

Accoring to the results of the study, NT-ProBNP proves that it might be useful in detecting patients with a bad prognosis, the analyzed enzyme having the capacity of identifying polytrauma patients with thoracic trauma that have high risk of death in the first 24 hours after admission.
\end{abstract}

Keywords: natriuretic peptide brain, NT-proBNP, polytrauma, thoracic trauma, emergency medicine

\section{Introduction}

Polytrauma and, in particular, thoracic trauma represent major emergencies, with high mortality risk, especially in younger population (1). The risk of death for patients experiencing severe trauma is high, approximately half them die in prehospital, and with high death rates in the first 24 to 72 hours after hospitalization (2).

Thoracic trauma is one of the main causes of death in polytrauma patients together with abdominal and head trauma (3). Also, studies revealed the fact that patients with polytrauma that associate chest trauma have higher death rates compared to polytrauma patients without chest trauma (4).

We investigated the 24 hours prognostic value of NT-proB-type Natriuretic Peptide (Nt-ProBNP) in polytraumatised patients with thoracic trauma.

\section{Material and Method}

We studied 33 patients with severe thoracic polytrauma, admitted through the emergency room of County Clinical Emergency Hospital Constanta between 2014 and 2017.

Inclusion criteria were age - over 16 years old, diagnosis of major polytrauma with thoracic 
trauma. We excluded from the study patients for whom information about the mechanism of trauma was unknown of a medical background was impossible to obtain.

For the purpose of the study, we measured, at the time of admission, NT-ProBNP levels. The endpoint of the study was considered mortality, within 24 hours from admission.

We used Matlab to calculate the accuracy of NT-ProBNP into identifying patients with very high risk of death in the next 24 hours. We decided to report the results by means of describing the receiver operating characteristic curve (ROC curve), which offers the sensitivity and sensibility for each cut point we obtained, and also we used the contingency table to report the results according to values that are specified in the medical literature (5).

\section{Results}

The study group was composed of 33 patients, 25 males and 8 females. During the study, the endpoint, which was death in the first 24 hours after admission was observed in 33\% of the patients (11 patients).

For the survivors group, the average age was 48.73 years, with a standard deviation of 14.01 years and median of 50 years, while for the patients that died, the average age was 50.64 , standard deviation 12.37 and median of 57 years. No statistically significant difference between groups was observed $(p=0.7)$.

Gender comparison between the two groups (patients that survived vs patients that died in the first 24 hours after admission) showed no statistically significant difference according to gender or living environment (Table I).

The Gaslgow Coma Score (GCS) was significantly lower in patients that died, with an average of 5.91, standard deviation of 4.56 and median of 3, compared to patients that survived, with an average of 12.45 , standard deviation of 3.82 and median of 14.5 .

NT-ProBNP values were significantly lower in the survival group, with an average of $203.23 \mathrm{pg} / \mathrm{ml}$, standard deviation of 223.28 $\mathrm{pg} / \mathrm{ml}$ and median of $80 \mathrm{pg} / \mathrm{ml}$, compared to the deceased group where average value was $854.91 \mathrm{pc} / \mathrm{ml}$, standard deviation $1845.12 \mathrm{pg} /$ $\mathrm{ml}$ and median of $300 \mathrm{pg} / \mathrm{ml}$. Because of the extreme values observed, for comparison we used logarithmic transformation. In Figure 1 we represent the distribution of NT-ProBNP values after logarithmic transformation.

Table I Baseline Characteristics of the Participants

\begin{tabular}{|l|c|c|c|}
\hline & $\begin{array}{c}\text { Survived } \\
(\mathbf{n}=\mathbf{2 2})\end{array}$ & Died (n=11) & $\mathbf{p}$ \\
\hline Age (years) & $\begin{array}{c}48.73 \\
(\mathrm{sd}=14.011)\end{array}$ & $\begin{array}{c}50.64 \\
(\mathrm{sd} 12.372)\end{array}$ & 0.7 \\
\hline Gender (Males) & $\begin{array}{c}72.7 \% \\
(\mathrm{n}=16)\end{array}$ & $81.8 \%(\mathrm{n}=9)$ & 0.68 \\
\hline $\begin{array}{l}\text { Environment } \\
\text { (Urban) }\end{array}$ & $\begin{array}{c}63.6 \% \\
(\mathrm{n}=14)\end{array}$ & $63.6 \%(\mathrm{n}=7)$ & 1 \\
\hline $\begin{array}{l}\text { Glasgow Coma } \\
\text { Score }\end{array}$ & $\begin{array}{c}12.45 \\
(\mathrm{sd}=3.82)\end{array}$ & $\begin{array}{c}5.91 \\
(\mathrm{sd}=4.65)\end{array}$ & 0.001 \\
\hline $\begin{array}{l}\text { NT-ProBNP } \\
(\mathrm{pg} / \mathrm{ml})\end{array}$ & $\begin{array}{c}203.23 \\
(\mathrm{sd}=223.38)\end{array}$ & $\begin{array}{c}854.91 \\
(\mathrm{sd}=1845.18)\end{array}$ & 0.028 \\
\hline
\end{tabular}

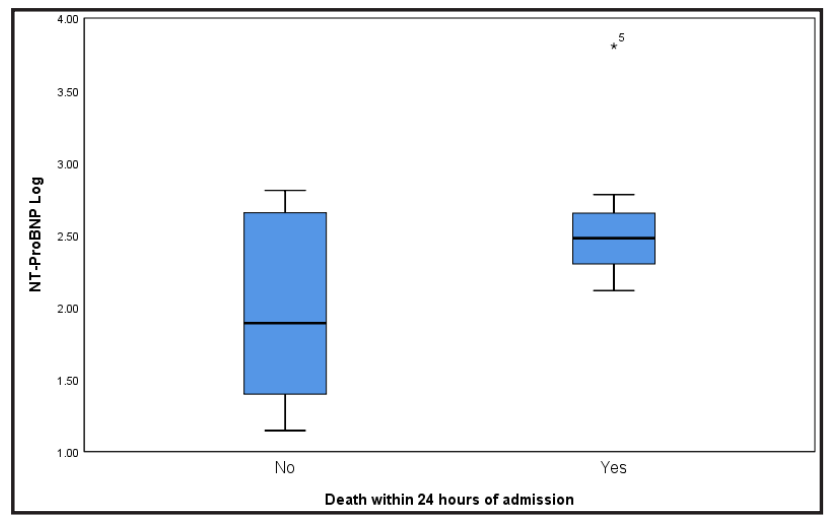

Figure 1 Distribution of the patients according to NTProBNP values

In order to determine the best cut-off points for positivity of NT-ProBNP values, we calculated the area under ROC curve, which was 0.738 with a $95 \%$ confidence interval of $0.556-$ 0.875 , this being statistically significant different from the 0.5 curve $(\mathrm{p}=0.0062)$ (Table II).

Table II Area under ROC curve

\begin{tabular}{|l|r|}
\hline Area under ROC curve & 0.738 \\
\hline Standard Error & 0.0869 \\
\hline Confidence interval 95\% & 0.556 to 0.875 \\
\hline Z statistic & 0.0062 \\
\hline Significance level P (Area=0.5) & 0.006 \\
\hline
\end{tabular}

Youden index, which is used for determining the best criterion to use based on the available data is 0.5909 for a cut-off positivity value of $120 \mathrm{pg} / \mathrm{ml}$. For this value, the sensitivity 
of the test is $100 \%$ and the specificity is $59.09 \%$ (Table III).

Table III Youden index $J$

\begin{tabular}{|l|r|}
\hline Youden index J & 0.5909 \\
\hline Associated criterion & $>120$ \\
\hline Sensitivity & 100.00 \\
\hline Specificity & 59.09 \\
\hline
\end{tabular}

The graphical representation of the ROC curve (Figure 2) shows the criterion points for which sensitivity and specificity (Table IV) were calculated.

Best sensitivity scores (100\%) were obtained for criterions of less than $120 \mathrm{pg} / \mathrm{ml}$, while best specificity scores were obtained for values higher than $600 \mathrm{pg} / \mathrm{ml}$.

Table IV NT-ProBNP performance

\begin{tabular}{|c|c|c|c|c|c|c|c|c|c|c|c|c|}
\hline Criterion & Sensitivity & $95 \% \mathrm{CI}$ & Specificity & $95 \% \mathrm{CI}$ & $+\mathrm{LR}$ & $95 \%$ CI & -LR & $95 \% \mathrm{CI}$ & $+\mathrm{PV}$ & $95 \%$ CI & -PV & $95 \% \mathrm{CI}$ \\
\hline$\geq 14$ & 100.00 & $\begin{array}{l}71.5- \\
100.0\end{array}$ & 0.00 & $\begin{array}{l}0.0- \\
15.4\end{array}$ & 1.00 & $1.0-1.0$ & & & 33.3 & $\begin{array}{c}18.0- \\
51.8\end{array}$ & & \\
\hline$>14$ & 100.00 & $\begin{array}{l}71.5- \\
100.0\end{array}$ & 4.55 & $\begin{array}{l}0.1- \\
22.8 \\
\end{array}$ & 1.05 & $1.0-1.1$ & 0.00 & & 34.4 & $\begin{array}{c}18.6- \\
53.2\end{array}$ & 100.0 & $\begin{array}{c}2.5- \\
100.0 \\
\end{array}$ \\
\hline$>15$ & 100.00 & $\begin{array}{l}71.5- \\
100.0 \\
\end{array}$ & 9.09 & $\begin{array}{l}1.1- \\
29.2 \\
\end{array}$ & 1.10 & $1.0-1.3$ & 0.00 & & 35.5 & $\begin{array}{c}19.2- \\
54.6\end{array}$ & 100.0 & $\begin{array}{l}15.8- \\
100.0\end{array}$ \\
\hline$>20$ & 100.00 & $\begin{array}{l}71.5- \\
100.0\end{array}$ & 18.18 & $\begin{array}{l}5.2- \\
40.3\end{array}$ & 1.22 & $1.0-1.5$ & 0.00 & & 37.9 & $\begin{array}{c}20.7- \\
57.7\end{array}$ & 100.0 & $\begin{array}{l}39.8- \\
100.0\end{array}$ \\
\hline$>25$ & 100.00 & $\begin{array}{l}71.5- \\
100.0\end{array}$ & 27.27 & $\begin{array}{c}10.7- \\
50.2\end{array}$ & 1.37 & $1.1-1.8$ & 0.00 & & 40.7 & $\begin{array}{c}22.4- \\
61.2 \\
\end{array}$ & 100.0 & $\begin{array}{l}54.1- \\
100.0 \\
\end{array}$ \\
\hline$>40$ & 100.00 & $\begin{array}{l}71.5- \\
100.0\end{array}$ & 31.82 & $\begin{array}{c}13.9- \\
54.9\end{array}$ & 1.47 & $1.1-2.0$ & 0.00 & & 42.3 & $\begin{array}{c}23.4- \\
63.1\end{array}$ & 100.0 & $\begin{array}{l}59.0- \\
100.0\end{array}$ \\
\hline$>50$ & 100.00 & $\begin{array}{l}71.5- \\
100.0\end{array}$ & 40.91 & $\begin{array}{c}20.7- \\
63.6 \\
\end{array}$ & 1.69 & $1.2-2.4$ & 0.00 & & 45.8 & $\begin{array}{c}25.6- \\
67.2\end{array}$ & 100.0 & $\begin{array}{l}66.4- \\
100.0 \\
\end{array}$ \\
\hline$>60$ & 100.00 & $\begin{array}{l}71.5- \\
100.0 \\
\end{array}$ & 50.00 & $\begin{array}{c}28.2- \\
71.8 \\
\end{array}$ & 2.00 & $1.3-3.0$ & 0.00 & & 50.0 & $\begin{array}{c}28.2- \\
71.8 \\
\end{array}$ & 100.0 & $\begin{array}{l}71.5- \\
100.0 \\
\end{array}$ \\
\hline$>100$ & 100.00 & $\begin{array}{l}71.5- \\
100.0\end{array}$ & 54.55 & $\begin{array}{c}32.2- \\
75.6\end{array}$ & 2.20 & $1.4-3.5$ & 0.00 & & 52.4 & $\begin{array}{c}29.8- \\
74.3\end{array}$ & 100.0 & $\begin{array}{l}73.5- \\
100.0\end{array}$ \\
\hline$>120$ & 100.00 & $\begin{array}{l}71.5- \\
100.0\end{array}$ & 59.09 & $\begin{array}{c}36.4- \\
79.3 \\
\end{array}$ & 2.44 & $1.5-4.0$ & 0.00 & & 55.0 & $\begin{array}{c}31.5- \\
76.9 \\
\end{array}$ & 100.0 & $\begin{array}{l}75.3- \\
100.0\end{array}$ \\
\hline$>130$ & 90.91 & $\begin{array}{c}58.7- \\
99.8\end{array}$ & 59.09 & $\begin{array}{c}36.4- \\
79.3\end{array}$ & 2.22 & $1.3-3.8$ & 0.15 & $0.02-1.0$ & 52.6 & $\begin{array}{c}28.9- \\
75.6\end{array}$ & 92.9 & $\begin{array}{l}66.1- \\
99.8\end{array}$ \\
\hline$>134$ & 81.82 & $\begin{array}{c}48.2- \\
97.7\end{array}$ & 59.09 & $\begin{array}{c}36.4- \\
79.3\end{array}$ & 2.00 & $1.1-3.6$ & 0.31 & $0.08-1.1$ & 50.0 & $\begin{array}{c}26.0- \\
74.0\end{array}$ & 86.7 & $\begin{array}{c}59.5- \\
98.3\end{array}$ \\
\hline$>150$ & 81.82 & $\begin{array}{c}48.2- \\
97.7\end{array}$ & 63.64 & $\begin{array}{c}40.7- \\
82.8\end{array}$ & 2.25 & $1.2-4.2$ & 0.29 & $0.08-1.0$ & 52.9 & $\begin{array}{c}27.8- \\
77.0\end{array}$ & 87.5 & $\begin{array}{c}61.7- \\
98.4\end{array}$ \\
\hline$>180$ & 72.73 & $\begin{array}{c}39.0- \\
94.0\end{array}$ & 63.64 & $\begin{array}{c}02.0 \\
40.7- \\
82.8\end{array}$ & 2.00 & $1.0-3.9$ & 0.43 & $0.2-1.2$ & 50.0 & $\begin{array}{c}24.7- \\
75.3\end{array}$ & 82.4 & $\begin{array}{c}56.6- \\
96.2\end{array}$ \\
\hline$>190$ & 72.73 & $\begin{array}{c}39.0- \\
94.0\end{array}$ & 68.18 & $\begin{array}{c}45.1- \\
86.1 \\
\end{array}$ & 2.29 & $1.1-4.7$ & 0.40 & $0.1-1.1$ & 53.3 & $\begin{array}{c}26.6- \\
78.7 \\
\end{array}$ & 83.3 & $\begin{array}{c}58.6- \\
96.4 \\
\end{array}$ \\
\hline$>220$ & 54.55 & $\begin{array}{c}23.4- \\
83.3\end{array}$ & 68.18 & $\begin{array}{c}45.1- \\
86.1\end{array}$ & 1.71 & $0.8-3.9$ & 0.67 & $0.3-1.4$ & 46.2 & $\begin{array}{c}19.2- \\
74.9\end{array}$ & 75.0 & $\begin{array}{l}50.9- \\
91.3\end{array}$ \\
\hline$>300$ & 45.45 & $\begin{array}{c}16.7- \\
76.6\end{array}$ & 72.73 & $\begin{array}{c}49.8- \\
89.3\end{array}$ & 1.67 & $0.7-4.3$ & 0.75 & $0.4-1.4$ & 45.5 & $\begin{array}{c}16.7- \\
76.6\end{array}$ & 72.7 & $\begin{array}{c}49.8- \\
89.3\end{array}$ \\
\hline$>320$ & 36.36 & $\begin{array}{c}10.9- \\
69.2\end{array}$ & 72.73 & $\begin{array}{c}49.8- \\
89.3\end{array}$ & 1.33 & $0.5-3.8$ & 0.88 & $0.5-1.5$ & 40.0 & $\begin{array}{c}12.2- \\
73.8\end{array}$ & 69.6 & $\begin{array}{c}47.1- \\
86.8\end{array}$ \\
\hline$>400$ & 27.27 & $\begin{array}{l}6.0- \\
61.0\end{array}$ & 72.73 & $\begin{array}{c}49.8- \\
89.3\end{array}$ & 1.00 & $0.3-3.3$ & 1.00 & $0.6-1.6$ & 33.3 & $\begin{array}{l}7.5- \\
70.1\end{array}$ & 66.7 & $\begin{array}{c}44.7- \\
84.4\end{array}$ \\
\hline$>450$ & 27.27 & $\begin{array}{l}6.0- \\
61.0 \\
\end{array}$ & 77.27 & $\begin{array}{c}54.6- \\
92.2 \\
\end{array}$ & 1.20 & $0.3-4.1$ & 0.94 & $0.6-1.4$ & 37.5 & $\begin{array}{l}8.5- \\
75.5 \\
\end{array}$ & 68.0 & $\begin{array}{c}46.5- \\
85.1 \\
\end{array}$ \\
\hline$>490$ & 27.27 & $\begin{array}{l}6.0- \\
61.0\end{array}$ & 81.82 & $\begin{array}{c}59.7- \\
94.8 \\
\end{array}$ & 1.50 & $0.4-5.6$ & 0.89 & $0.6-1.3$ & 42.9 & $\begin{array}{l}9.9- \\
81.6 \\
\end{array}$ & 69.2 & $\begin{array}{c}48.2- \\
85.7 \\
\end{array}$ \\
\hline$>500$ & 18.18 & $\begin{array}{l}2.3- \\
51.8 \\
\end{array}$ & 86.36 & $\begin{array}{c}65.1- \\
97.1 \\
\end{array}$ & 1.33 & $0.3-6.8$ & 0.95 & $0.7-1.3$ & 40.0 & $\begin{array}{l}5.3- \\
85.3 \\
\end{array}$ & 67.9 & $\begin{array}{c}47.6- \\
84.1 \\
\end{array}$ \\
\hline$>550$ & 18.18 & $\begin{array}{l}2.3- \\
51.8\end{array}$ & 90.91 & $\begin{array}{l}70.8- \\
98.9\end{array}$ & 2.00 & $0.3-12.4$ & 0.90 & $0.7-1.2$ & 50.0 & $\begin{array}{l}6.8- \\
93.2\end{array}$ & 69.0 & $\begin{array}{c}49.2- \\
84.7\end{array}$ \\
\hline$>600$ & 9.09 & $\begin{array}{l}0.2- \\
41.3\end{array}$ & 95.45 & $\begin{array}{c}77.2 \text { - } \\
99.9\end{array}$ & 2.00 & $0.1-29.0$ & 0.95 & $0.8-1.2$ & 50.0 & $\begin{array}{l}1.3- \\
98.7\end{array}$ & 67.7 & $\begin{array}{c}48.6- \\
83.3\end{array}$ \\
\hline$>642$ & 9.09 & $\begin{array}{l}0.2- \\
41.3 \\
\end{array}$ & 100.00 & $\begin{array}{l}84.6- \\
100.0\end{array}$ & & & 0.91 & $0.8-1.1$ & 100.0 & $\begin{array}{c}2.5- \\
100.0\end{array}$ & 68.8 & $\begin{array}{c}50.0- \\
83.9\end{array}$ \\
\hline$>6400$ & 0.00 & $\begin{array}{l}0.0- \\
28.5\end{array}$ & 100.00 & $\begin{array}{l}84.6- \\
100.0\end{array}$ & & & 1.00 & $1.0-1.0$ & & & 66.7 & $\begin{array}{c}48.2- \\
82\end{array}$ \\
\hline
\end{tabular}




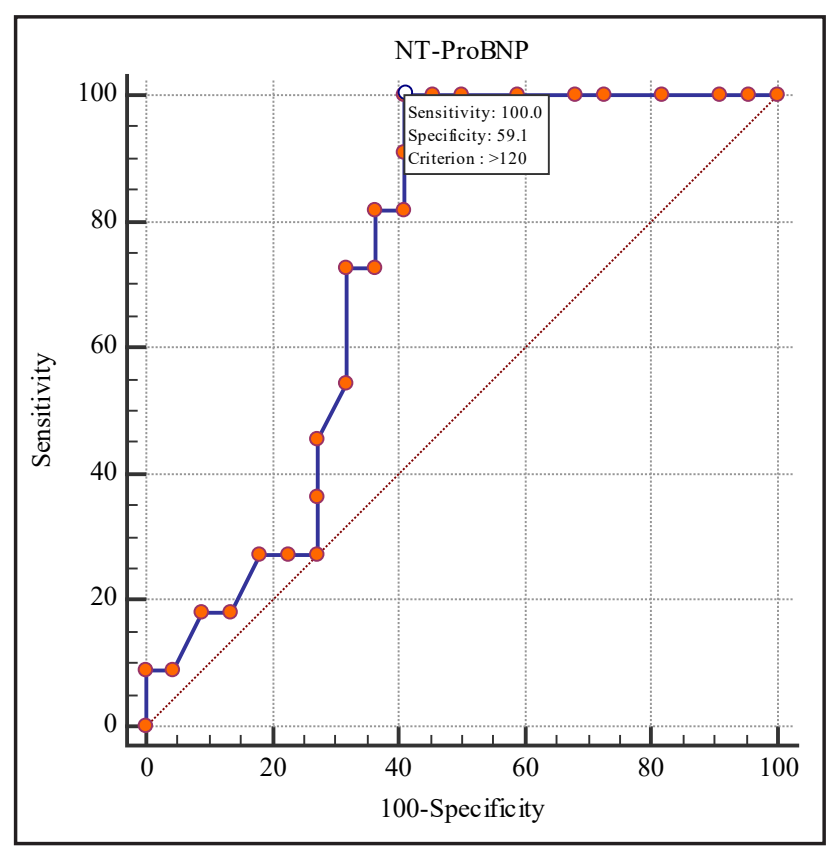

Figure 2 ROC curve for NT-ProBNP

Table V Crosstabultation for death within 24 hours of admission and NT-ProBNP category (cut-off point 125 $\mathrm{pg} / \mathrm{ml}$ )

\begin{tabular}{|c|c|c|c|c|c|}
\hline & \multicolumn{2}{|c|}{$\begin{array}{l}\text { Death within } \\
24 \text { hours of } \\
\text { admission }\end{array}$} & \multirow[b]{2}{*}{ Total } \\
\hline & & & No & Yes & \\
\hline \multirow{4}{*}{$\begin{array}{l}\text { NT- } \\
\text { ProBNP }\end{array}$} & \multirow[b]{2}{*}{$\begin{array}{l}\text { Normal } \\
(\leq 125 \\
\mathrm{pg} / \mathrm{ml})\end{array}$} & Count & 13 & 0 & 13 \\
\hline & & $\begin{array}{l}\text { \% within } \\
\text { Death within } \\
24 \text { hours of } \\
\text { admission }\end{array}$ & $59.1 \%$ & $0.0 \%$ & $39.4 \%$ \\
\hline & \multirow{2}{*}{\begin{tabular}{|l} 
Elevated \\
$(>125$ \\
$\mathrm{pg} / \mathrm{ml})$
\end{tabular}} & Count & 9 & 11 & 20 \\
\hline & & $\begin{array}{l}\text { \% within } \\
\text { Death within } \\
24 \text { hours of } \\
\text { admission }\end{array}$ & $40.9 \%$ & $100.0 \%$ & $60.6 \%$ \\
\hline \multirow{2}{*}{\multicolumn{2}{|c|}{$\begin{array}{l}\text { Total } \\
\% \text { within Death } \\
\text { within } 24 \text { hours of } \\
\text { admission }\end{array}$}} & Count & 22 & 11 & 33 \\
\hline & & $100.0 \%$ & $100.0 \%$ & $100.0 \%$ & \\
\hline
\end{tabular}

Using a cut-off point of $125 \mathrm{pg} / \mathrm{ml}$, the sensitivity of the test was $100 \%$ and the specificity was $59.09 \%$. Complete analysis - Table VI.
Table VI Test accuracy for NT-ProBNP cut-off point value of $125 \mathrm{pg} / \mathrm{ml}$

\begin{tabular}{|c|c|c|}
\hline & Value & CI 95\% \\
\hline Sensitivity & $100.00 \%$ & $71.51 \%$ to $100.00 \%$ \\
\hline Specificity & $59.09 \%$ & $36.35 \%$ to $79.29 \%$ \\
\hline AUC & 0.80 & 0.62 to 0.92 \\
\hline $\begin{array}{l}\text { Positive } \\
\text { Likelihood Ratio }\end{array}$ & 2.44 & 1.48 to 4.04 \\
\hline $\begin{array}{l}\text { Negative } \\
\text { Likelihood Ratio }\end{array}$ & 0.00 & \\
\hline Disease prevalence & $33.33 \%$ & $17.96 \%$ to $51.83 \%$ \\
\hline $\begin{array}{l}\text { Positive } \\
\text { Predictive Value }\end{array}$ & $55.00 \%$ & $31.53 \%$ to $76.94 \%$ \\
\hline $\begin{array}{l}\text { Negative } \\
\text { Predictive Value }\end{array}$ & $100.00 \%$ & $75.29 \%$ to $100.00 \%$ \\
\hline
\end{tabular}

\section{Discussion}

The results offer an important perspective on using it for initial evaluation of the patients. The results indicate that a cut-off point of $125 \mathrm{pg} /$ $\mathrm{ml}$ is suitable for the test, having the capacity of correctly identifying patients at risk of $100 \%$ and of correctly identifying patients with low risk of $59.09 \%$.

Studies proved that NT-ProBNP levels correlate with cardiac impairment (7) in patients with multiple injuries. Also, in a study from 2015 (8) the authors concluded that NT-ProBNP analysis might offer additional information for the diagnosis of cardiac traumatisms in the initial evaluation of patients with thoracic trauma.

Other studies analyzed the importance of determining NT-ProBNP values in patients that undergone thoracic surgery (9-11), patients with heart failure (12) or in patients that undergo surgery for abdominal aneurisms (13).

Limitations of this study are related especially to the unknown cardiac status of the patients before hospital admission, therefore the impossibility of determining if the increase of NT-ProBNP values in some of them are because of the induced trauma or because of a preexisting condition.

\section{Conclusions}

Accoring to the results of the study, NTProBNP proves that it might be useful in detecting patients with a bad prognosis, the analyzed enzyme having the capacity of identifying 
polytrauma patients with thoracic trauma that have high risk of death in the first 24 hours after admission.

\section{References}

1. Pape HC, Peitzman AB, Schwab CW, Giannoudis PV. Damage Control Management in the Polytrauma Patient: Springer New York; 2010.

2. Masella CA, Pinho VF, Costa Passos AD, Spencer Netto FA, Rizoli S, Scarpelini S. Temporal distribution of trauma deaths: quality of trauma care in a developing country. J Trauma. 2008;65(3):653-8.

3. Chrysou K, Halat G, Hoksch B, Schmid RA, Kocher GJ. Lessons from a large trauma center: impact of blunt chest trauma in polytrauma patients - still a relevant problem? Scandinavian Journal of Trauma, Resuscitation and Emergency Medicine. 2017;25:42.

4. Vécsei V, Arbes S, Aldrian S, Nau T. Chest Injuries in Polytrauma. European Journal of Trauma. 2005;31(3):239-43.

5. Bossuyt PM, Reitsma JB, Bruns DE, Gatsonis CA, Glasziou PP, Irwig L, et al. STARD 2015: an updated list of essential items for reporting diagnostic accuracy studies. BMJ. 2015;351:h5527.

6. Schisler JC, Lang $\mathrm{CH}$, Willis $\mathrm{M}$. Endocrinology of the Heart in Health and Disease: Integrated, Cellular, and Molecular Endocrinology of the Heart: Elsevier Science; 2016.

7. Kirchhoff C, Leidel BA, Kirchhoff S, Braunstein V, Bogner V, Kreimeier U, et al. Analysis of N-terminal pro-B-type natriuretic peptide and cardiac index in multiple injured patients: a prospective cohort study. Critical Care. 2008;12(5):R118-R.

8. Dogan H, Sarikaya S, Neijmann ST, Uysal E, Yucel N, Ozucelik DN, et al. $\mathrm{N}$-terminal pro-B-type natriuretic peptide as a marker of blunt cardiac contusion in trauma. International journal of clinical and experimental pathology. 2015;8(6):6786-92.

9. Lee CY, Bae MK, Lee JG, Kim K-W, Park IK, Chung KY. N-Terminal Pro-B-type Natriuretic Peptide Is Useful to Predict
Cardiac Complications Following Lung Resection Surgery. The Korean Journal of Thoracic and Cardiovascular Surgery. 2011;44(1):44-50.

10. Salvatici M, Cardinale D, Spaggiari L, Veglia F, Tedesco CC, Solli P, et al. Atrial fibrillation after thoracic surgery for lung cancer: use of a single cut-off value of N-terminal pro-B type natriuretic peptide to identify patients at risk. Biomarkers. 2010;15(3):259-65.

11. Cardinale D, Colombo A, Sandri MT, Lamantia G, Colombo N, Civelli M, et al. Increased perioperative N-terminal pro-Btype natriuretic peptide levels predict atrial fibrillation after thoracic surgery for lung cancer. Circulation. 2007;115(11):1339-44.

12. Kristensen SL, Jhund PS, Mogensen UM, Rorth R, Abraham WT, Desai A, et al. Prognostic Value of N-Terminal Pro-B-Type Natriuretic Peptide Levels in Heart Failure Patients With and Without Atrial Fibrillation. Circulation Heart failure. 2017;10(10).

13. Feringa HH, Bax JJ, Elhendy A, de Jonge R, Lindemans J, Schouten O, et al. Association of plasma N-terminal pro-B-type natriuretic peptide with postoperative cardiac events in patients undergoing surgery for abdominal aortic aneurysm or leg bypass. The American journal of cardiology. 2006;98(1):111-5. 